\title{
The Relationship of Dry Eye Disease With Depression in Saudi Arabia: A Cross-Sectional Study
}

\author{
Walaa Al-Dairi ${ }^{1}$, Omar M. AL Sowayigh ${ }^{2}$, Noura S. Alkulaib ${ }^{2}$, Ali Alsaad ${ }^{3}$ \\ 1. Surgery, College of Medicine, King Faisal University, AlAhsa, SAU 2. Ophthalmology, College of Medicine, King \\ Faisal University, AlAhsa, SAU 3. Psychiatry, College of Medicine, King Faisal University, AlAhsa, SAU
}

Corresponding author: Omar M. AL Sowayigh,omar-m-008@hotmail.com

\section{Abstract \\ Objective}

Estimate the prevalence of depression among individuals with a dry eye disease (DED) in Saudi Arabia using two questionnaires: Patient Health Questionnaire (PHQ-9) and Dry Eye Questionnaire (DEQ-5), and explore potential factors implicated in the development of depression among the DED population.

\section{Methods}

This is a descriptive cross-sectional study of 476 patients with DED which was conducted using a PHQ-9 questionnaire to screen for depression and a DEQ-5 questionnaire to diagnose DED. The questionnaires were merged and distributed using Google Forms through various social media platforms targeting the Saudi population. After data collection, it was revised, coded and fed to statistical software IBM SPSS version 22 (SPSS, Inc. Chicago, IL).

\section{Results}

Depression was diagnosed among 200 participants (42\%) of the cases with dry eyes. From which $5.7 \%$ had mild depression, $13.9 \%$ had moderate depression, $12.6 \%$ had moderately severe depression, and $9.9 \%$ had severe depression. A female predominance was noticed; $44.7 \%$ of the females with dry eyes had depression compared to $32.4 \%$ of males with recorded statistical significance ( $\mathrm{P}=0.023)$. Depression was detected among $55 \%$ of those who are less than 20 years old in comparison to $27 \%$ of those who are 30 years or older $(\mathrm{P}=0.001)$. Laser-assisted in-situ keratomileusis (LASIK), prolonged electronic device usage and contact lens wear are reported as risk factors associated with an increase in dry eye symptoms. However, there is no statistically significant relationship between contact lens wear and depression among dry eye disease patients.

\section{Conclusion}

Review began $12 / 09 / 2020$ Review ended 12/11/2020 Published 12/18/2020

(c) Copyright 2020

Al-Dairi et al. This is an open access article distributed under the terms of the Creative Commons Attribution License CC-BY 4.0., which permits unrestricted use, distribution, and reproduction in any medium, provided the original author and source are credited.
Suffering from DED is a possible risk factor for developing depression as DED is associated with depression of higher degrees of severity. Depression among DED patients is found to be significantly more prevalent among females and the young adult population rather than older adults.

Categories: Ophthalmology, Psychiatry

Keywords: dry eye disease, dry eye disorder, depression, patient health questionnaire (phq-9), dry eye questionnaire (deq-5), contact lens

\section{Introduction}

Dry eye disease (DED) is a multifactorial disease that affects the tear film and ocular surface of the eye. DED leads to abnormal alterations of the normal ocular surface physiology including an increase in the osmolarity of the tear film and inflammation. As a result of these changes, patients with dry eyes complain of eye discomfort, irritation or burning sensation in addition to visual disturbances. Furthermore, tear film instability is noted on tear breakup time with potential damage to the ocular surface [1].

DED has a worldwide prevalence of 5-50\% [2]. In Saudi Arabia, the prevalence is high yet highly variable, which is owed to the different studied provinces and cities ranging from $32.1 \%$ to $93.2 \%$ [3-5]. Globally, DED is more prevalent among females [2,6], with a similar finding in Saudi Arabia [3]. Other than female gender predominance, risk factors include old age [7], blepharitis [5], smoking [5] and having a chronic disease such as diabetes mellitus, arthritis and hypercholesterolemia [7,8].

DED has a significant impact on both the patient's quality of life, noted by Short Form 36 Health Survey Questionnaire (SF-36) scores [9], and vision-related quality of life, noted by difficulties in reading, 
watching television, conducting professional work, computer use and driving [10]. Furthermore, DED negatively influences psychological health, and in effect, is associated with developing depression [11,12]. Depression is a serious, however, overlooked mental health illness that negatively affects the mood and is potentially life-threatening if neglected. Although the etiology is still poorly understood, numerous factors and diseases have been linked to or resulted in depression.

Among other eye diseases, DED was found to be the only disease to have an increased depression score using Zung's self-rated depression scale [12]. Many studies in the literature have found a higher prevalence of depression among DED patients compared to controls [13] and a linear association between DED and depressive symptoms [14] which suggests a higher risk among chronic severe DED patients [11,14]. The visual impairment caused by dry eye symptoms is directly linked to depressive symptoms [15]. Furthermore, a reverse relationship was reported as well, in which depression was associated with DED [16] and worsening of DED symptoms [14]. In this respect, DED and depression have a manifest interrelation in ways that have a negative impact on the patients [17].

Depression screening questionnaires and self-rating scales such as the nine-item Patient Health Questionnaire (PHQ-9) and Zung's self-rated depression scale have been useful in primary care settings and research purposes $[18,19]$. These scales have also been used in studies evaluating the relationship between certain eye diseases and depression $[12,20]$. Applying the same concept, the Dry Eye Questionnaire (DEQ-5) has been used in the diagnosis of DED with quantification of its severity level [21,22].

Despite the high prevalence of both DED and depression in Saudi Arabia [3-5,23], the psychological impact of DED has not been studied adequately. Therefore, this study aims to evaluate the relationship between DED and depression in Saudi Arabia using two questionnaires: PHQ-9 and DEQ-5.

\section{Materials And Methods Study design and participants}

This descriptive cross-sectional study was conducted using a PHQ-9 questionnaire to screen for depression and a DEQ-5 questionnaire to diagnose DED. DEQ- 5 was translated into Arabic for easy accessibility to the targeted population (Appendices). A unified single questionnaire was distributed using Google Forms through various social media platforms targeting Saudi individuals.

This study included participants who are aged 15 and older, who are either previously diagnosed with dry eyes or had DED based on DEQ-5. For exclusion criteria, all individuals who were previously diagnosed with depression or suffering from any other psychiatric illness, have more than one comorbid condition (e.g., heart disease, hypertension, diabetes mellitus, asthma, etc.), were grieving the death of a close person, or had a recent ocular procedure done in less than a year including laser-assisted in-situ keratomileusis (LASIK), crosslinking or corneal transplantation were excluded. Among 1568 participants, only 476 (30.35\%) met the criteria and were included for analysis.

Other data include biographical information such as age, gender, province/city, eye conditions, eye procedures, daily screen time, and contact lens use.

\section{Statistical analysis}

After data were extracted, it was revised, coded, and fed to statistical software IBM SPSS version 22 (SPSS, Inc. Chicago, IL). All statistical analysis was done using two-tailed tests. A P-value less than 0.05 was statistically significant. Descriptive analysis based on frequency and percent distribution was done for patient's characteristics, eye care, and depression severity. Regarding PHQ-9, the total score for the different items was summed and categorized into no depression (zero score), minimal depression (1-4), mild depression (5-9), moderate depression (10-14), moderately severe depression (15-19), and severe depression (20-27). Cross-tabulation was used to assess the relationship between personal data and depression and between eye care and dry eye data with different items including contact lens use, daily screen time. Pearson chi-square and exact probability tests were used to test for distribution difference significance.

\section{Ethical consideration}

This study was conducted upon the approval of the research ethics committee of the College of Medicine, King Faisal University. The DEQ- 5 questionnaire was used with direct permission from Robin L Chalmers. All data were confidentially and anonymously collected with consent taken from the participants.

\section{Results}

This study included 476 cases with dry eyes whose ages ranged from 15 to 54 years old with a mean age of $22.7 \pm 6.8$ years old. The majority of the participants were females (77.9\%; 371) and 212 (44.5\%) work indoors with prolonged electronic screen using. Regarding regions, 155 cases (32.6\%) were from Riyadh and 70 (14.7\%) were from Al-Ahsa. Over 235 cases (49.4\%) were previously diagnosed with dry eyes and 50.6\% were 


\section{Cureus}

diagnosed using the DEQ-5 scale. Also, 63 (13.2\%) had a chronic health problem (e.g., heart disease, hypertension, diabetes mellitus, asthma, etc.). As for other eye diseases, astigmatism was the most reported by the cases $(81.4 \%$; 79$)$, followed by myopia (76.3\%; 74), hyperopia (29.9\%; 29$)$, and amblyopia $(12.4 \% ; 12)$ while only three cases had glaucoma (3.1\%; Table 1$)$.

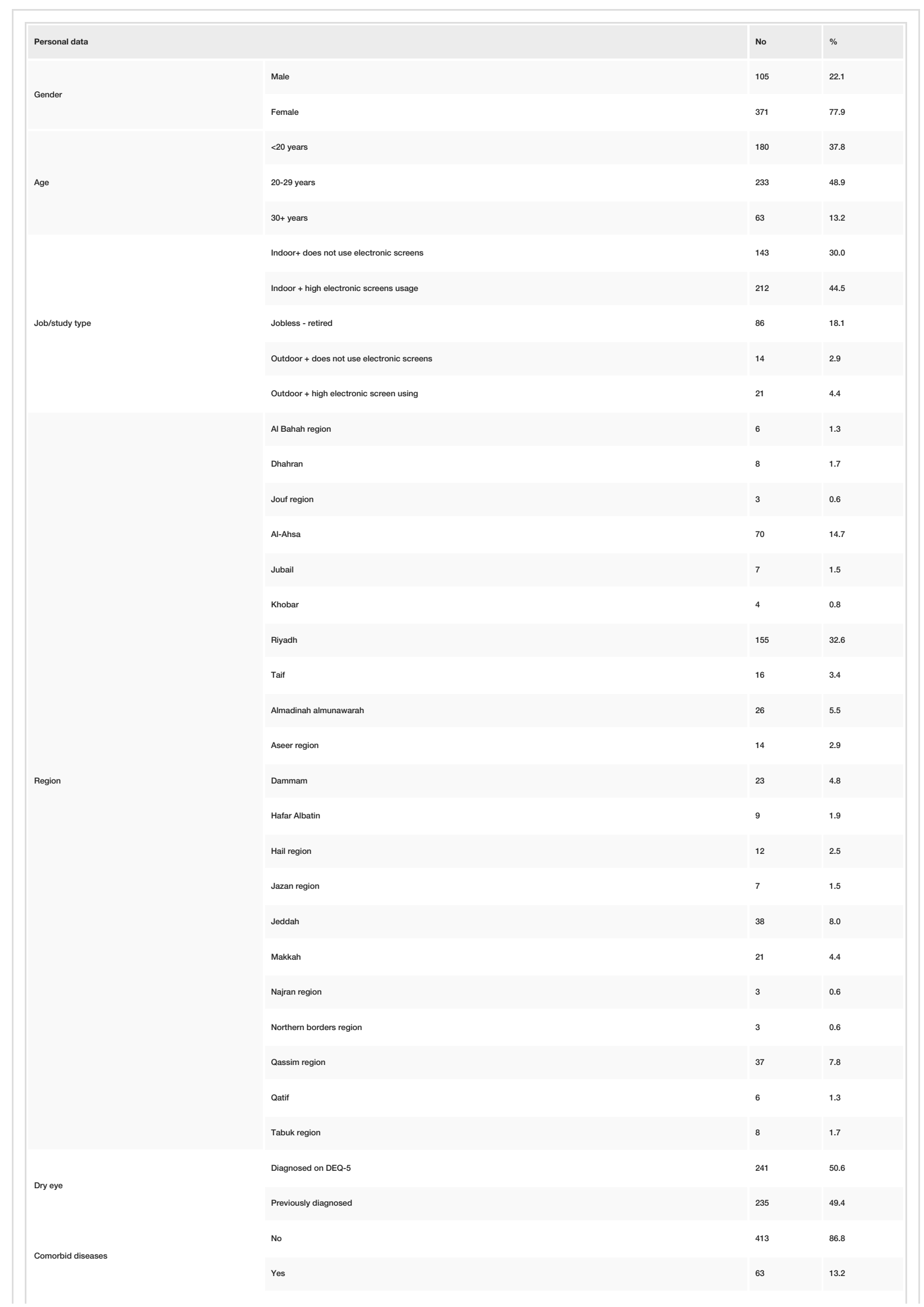




\section{Cureus}

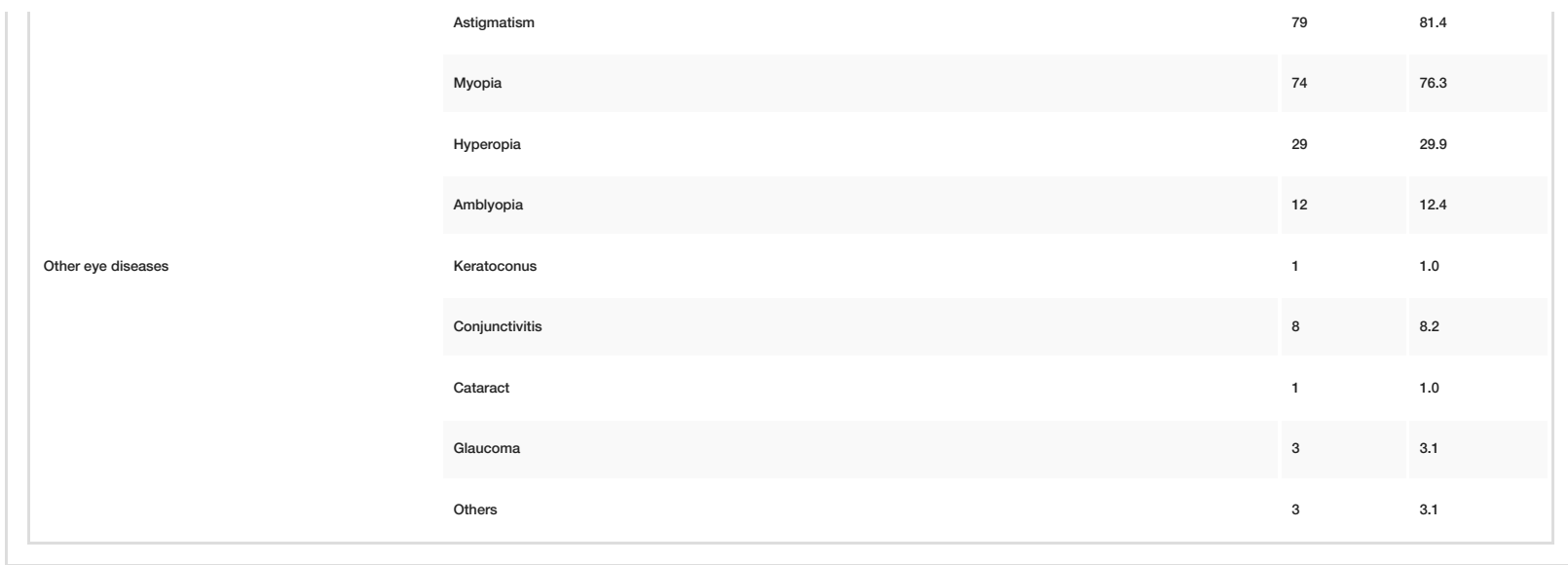

TABLE 1: Personal data of patients with DED, Saudi Arabia, 2020.

DED: dry eye disease, DEQ: Dry Eye Questionnaire.

Depression was diagnosed among 200 (42\%) of the cases with dry eyes, of which $5.7 \%$ had mild depression, 13.9\% had moderate depression, $12.6 \%$ had moderately severe depression, and $9.9 \%$ had severe depression (Table 2).

\begin{tabular}{|c|c|c|}
\hline Depression severity & No & $\%$ \\
\hline No depression & 276 & 58.0 \\
\hline Depression & 200 & 42.0 \\
\hline Mild depressi & 27 & 5.7 \\
\hline Moderate depression & 66 & 13.9 \\
\hline Moderately severe depression & 60 & 12.6 \\
\hline Severe depression & 47 & 9.9 \\
\hline
\end{tabular}

TABLE 2: Prevalence of depression and its severity among patients with dry eye disease.

Regarding gender predilection, $44.7 \%$ of females with DED had depression compared to $32.4 \%$ of males with recorded statistical significance $(\mathrm{P}=0.023)$. As for age, depression was detected among $55 \%$ of those who were less than 20 years of age in comparison to $27 \%$ of those who were 30 years or older $(\mathrm{P}=0.001)$.

Considering the geographical distribution of depression in our sample, there was percentage variability among the regions, however, a larger sample size would help to eliminate any statistical bias.

Considering other concurrent eye diseases, $66.7 \%$ of those with amblyopia had depression compared to $62.5 \%$ of those with conjunctivitis and $51.9 \%$ of those with astigmatism $(\mathrm{P}=0.379)$. An exact $42.6 \%$ of those with no health problem were depressed compared to $38.1 \%$ of those with chronic health problem with no statistical significance (Table 3).

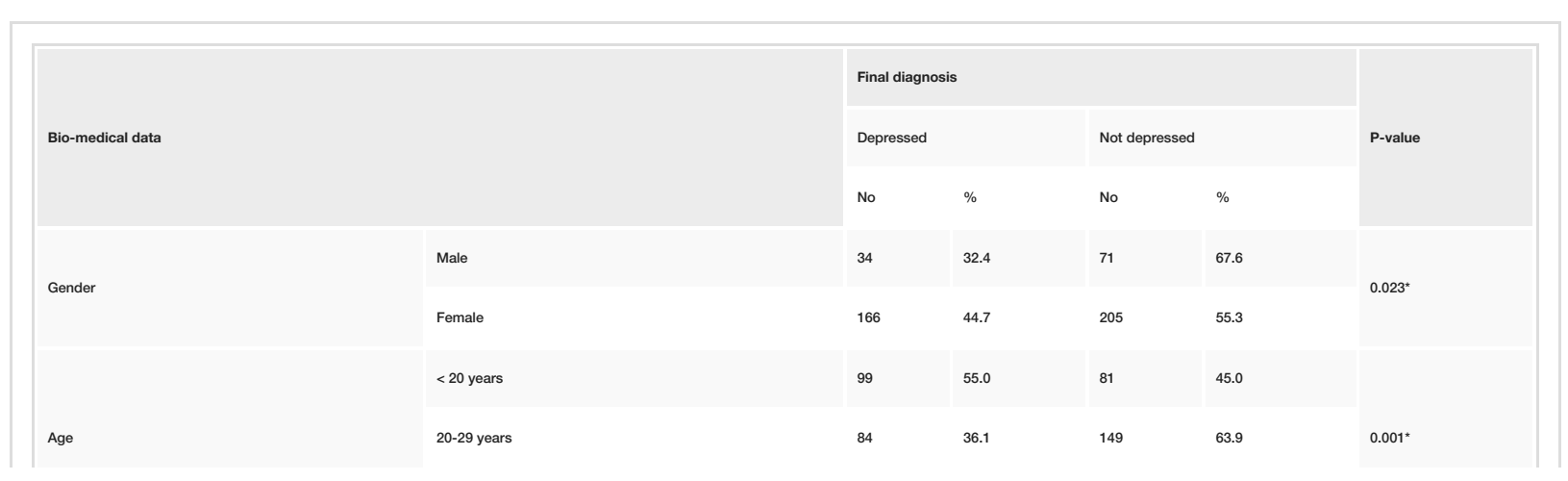




\section{Cureus}

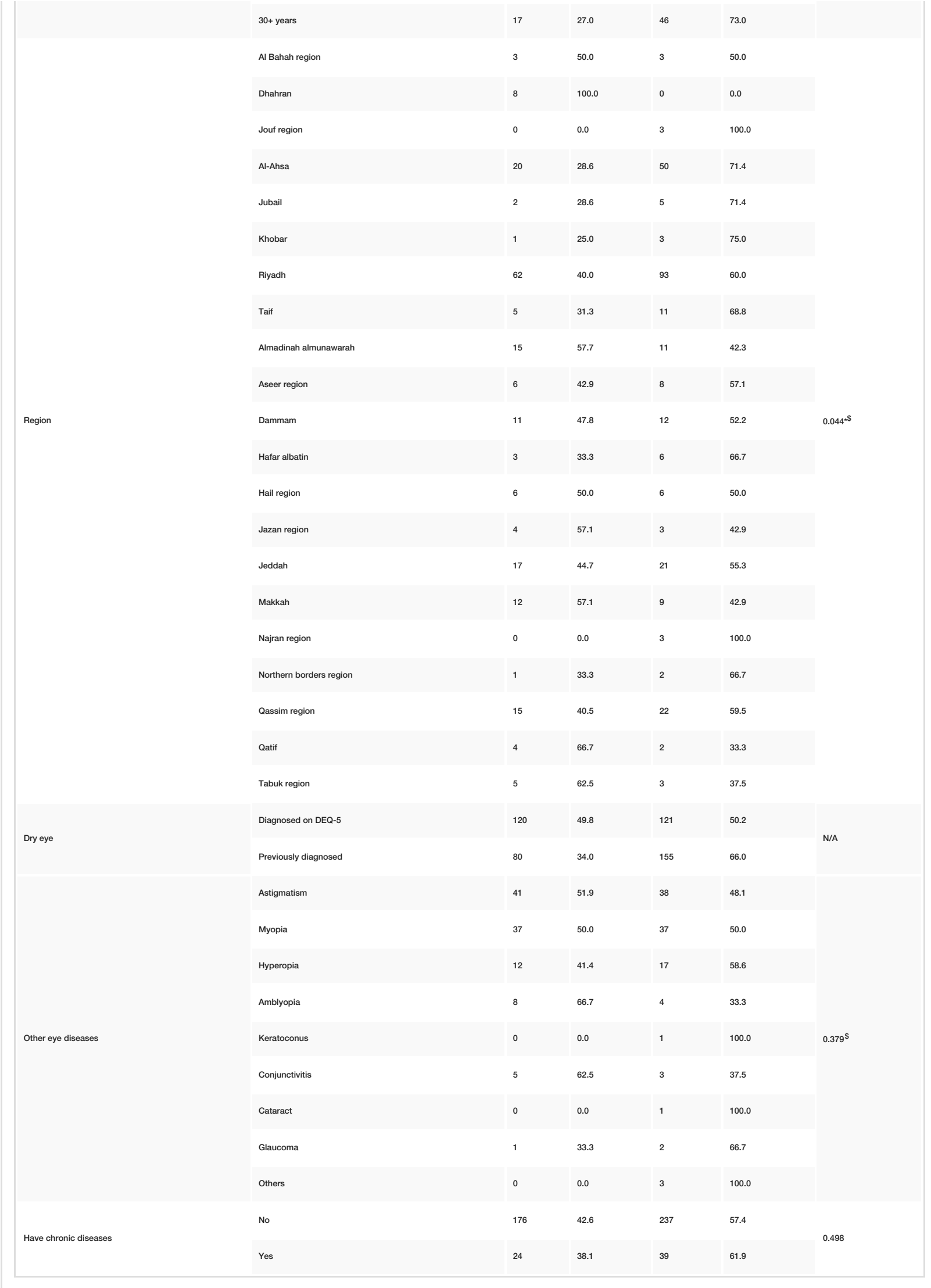

TABLE 3: Effect of different patients with dry eye disease characteristics and comorbidities on depression status.

P: Pearson X2 test, N/A: not applicable.

${ }^{*} \mathrm{P}<0.05$ (significant). 


\section{Cureus}

\$Exact probability test.

Table 4 illustrates the daily screen time and history of eye procedures among patients with dry eye. A small percentage (12.6\%) recorded daily screen time of less than five hours while $33.2 \%$ recorded 10 hours or more. An increase of dry eye symptoms associated with electronic device usage was reported by 413 (86.8\%) of the cases. With respect to ocular procedures, $7.8 \%$ had a procedure more than a year. LASIK surgery was the most reported procedure $(5.9 \% ; 28)$ followed by the chalazion procedure $(1.7 \% ; 8)$. Daily contact lens (CL) use was reported by 17 cases (3.6\%). An increase in dryness symptoms with CL use was reported by $42 \%$ of the cases while $44.6 \%$ reported that it may occur sometimes. The need of using artificial eye drops during CL wear was reported by 86 (76.8\%) of the cases, and 64 (58\%) became intolerant of CL use which pushed 53 $(82.8 \%)$ to use eyeglasses. 


\section{Cureus}

\begin{tabular}{|c|c|c|c|}
\hline Eye procedures and screen time data & & No & $\%$ \\
\hline & $<5$ hours & 60 & 12.6 \\
\hline Daily screen time & 5-9 hours & 258 & 54.2 \\
\hline & $10+$ hours & 158 & 33.2 \\
\hline & Yes & 413 & 86.8 \\
\hline & No & 63 & 13.2 \\
\hline & No & 434 & 91.2 \\
\hline Had an eye procedure & Yes, less than a year & 5 & 1.1 \\
\hline & Yes, more than a year & 37 & 7.8 \\
\hline & None & 434 & 91.2 \\
\hline & Chalazion procedure & 8 & 1.7 \\
\hline & LASIK & 28 & 5.9 \\
\hline & Punctual occlusion & 1 & 0.2 \\
\hline 年 & Removal of foreign object procedure & 1 & 0.2 \\
\hline & Retinal detachment procedure & 1 & 0.2 \\
\hline & Retinal procedure & 1 & 0.2 \\
\hline & Strabismus procedure & 2 & 0.4 \\
\hline & Daily & 17 & 3.6 \\
\hline & 1-2 days/ week & 14 & 2.9 \\
\hline Cluse & Sometimes & 81 & 17.0 \\
\hline & No & 364 & 76.5 \\
\hline & Yes & 47 & 42.0 \\
\hline Dryness increases with CL use & Sometimes & 50 & 44.6 \\
\hline & No & 15 & 13.4 \\
\hline & Yes & 86 & 76.8 \\
\hline & No & 26 & 23.2 \\
\hline & Yes & 64 & 58.0 \\
\hline 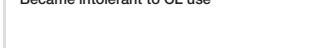 & No & 47 & 42.0 \\
\hline & Yes & 53 & 82.8 \\
\hline If yes, does it push you to use eyeglasses? & No & 11 & 17.2 \\
\hline
\end{tabular}

TABLE 4: Daily screen time and history of eye procedures among patients with dry eye disease.

CL: contact lens, LASIK: laser-assisted in-situ keratomileusis.

Depression was detected among $47.1 \%$ of those who use CL daily in comparison to $59.3 \%$ of those who did not use it ( $\mathrm{P}=0.481$; Table 5). Intolerance to $\mathrm{CL}$ developed in $52.3 \%$ of the wearers compared to $55.3 \%$ of those who were able to continue wearing them $(\mathrm{P}=0.752)$. Also, $52.8 \%$ of those who have dry eyes and used eyeglasses for refractive errors had depression compared to $45.5 \%$ of those who did not need to wear glasses $(\mathrm{P}=0.656)$. 


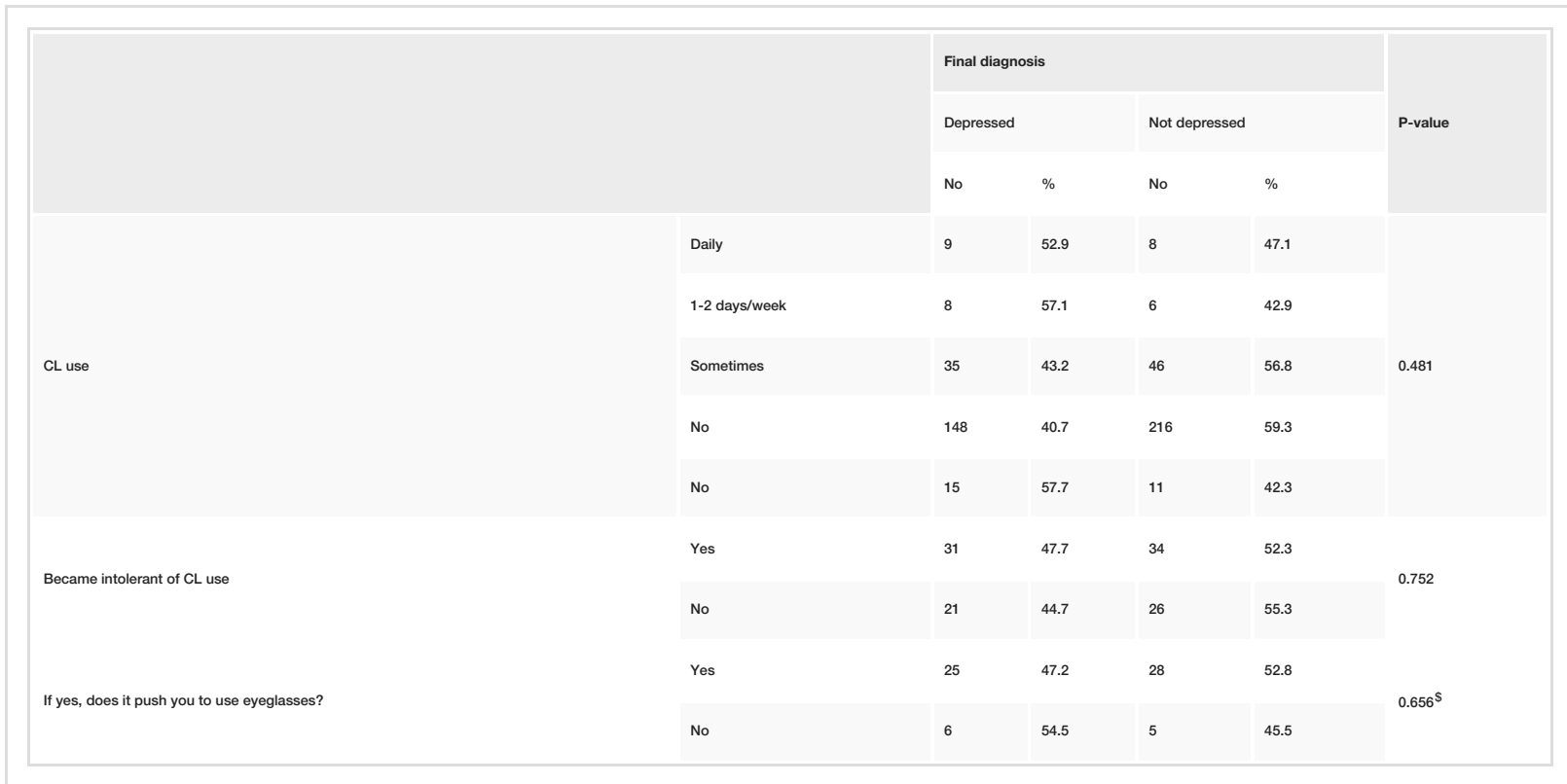

\section{TABLE 5: Distribution of depression among patients with dry eyes according to $C L$ use.}

$\mathrm{CL}$, contact lens, P: Pearson $\mathrm{X}^{2}$ test.

SEact probability test.

\section{Discussion}

To the best of our knowledge, this is the first study to explore the relationship between DED and depression in Saudi Arabia. Using PHQ-9 and DEQ-5 questionnaires, we were able to assess depression and DED among Saudi adults living in Saudi Arabia. We found that $42 \%$ of individuals suffering from dry eyes had depression with various degrees of severity. Those individuals were not previously diagnosed with depression or any psychiatric illness nor were in a grief state. By eliminating such conditions, we emphasized the impact of DED on the quality of life and mood.

Similar studies have explored the associations of DED, depression and anxiety. Most of these studies have proved a significant relationship [13,17,24]. According to the literature, the relationship seems to start either way, where DED plays a role in experiencing depression and vice versa [17]. This signifies the importance of detecting depression among individuals with DED as well as identifying the role of chronic conditions and their medications which could be the underlying cause for dry eyes among many individuals.

It has been previously established that depression is more prevalent among females $[2,6]$, which is a similar finding in this study where females with DED were significantly more likely to suffer from depression than males. We also found that young adults with DED tend to suffer from depression more than older adults. This is in accordance with other studies that found depression to be less common among older adults [25]. Although the depression rate among DED patients (42\%) is not higher than the rate reported in 2014 among the general Saudi adult population (49.9\%) [23], depression's severity is higher among DED patients.

Having a concurrent eye disease did not seem to have a significant relationship with developing depression. This is clearly evident in the investigated common ocular conditions like astigmatism, myopia, and hyperopia. However, a wider study scope with a larger inclusive sample will be needed to investigate the other ocular pathologies mentioned in our study which are: amblyopia, keratoconus, conjunctivitis, cataract, and glaucoma. Having a concomitant chronic disease seemed to has no effect on developing depression among individuals with DED. We assume that the direct impact of DED on the person's daily activity is the reason behind the tendency of developing depression by the affected individuals more than other chronic health conditions with subtle psychological effects.

Furthermore, Akkaya et al. [26] described a comparable finding to this study where DED symptoms increase with prolonged daily electronic device usage. Among the different eye procedures, LASIK and cataract surgeries were the most procedures known to result in transient DED as reported in the literature [27,28]. These findings point out the role of ocular procedures and individual's screen time in developing DED. LASIK surgery is complicated by DED mainly because of iatrogenic corneal nerve damage [29]. On the other hand, the use of electronic devices decreases the number of eye blinks and subsequently leads to dry eye 


\section{Cureus}

symptoms [26]. Hence, recognizing this fact should allow individuals to exercise a protective attitude against DED and its consequences by improving lifestyle, such as limiting screen time as much as possible, use of 20-20-20 rule to reduce the symptoms of eye strain and dryness associated with prolonged electronic device usage, enhancing the indoor humidity by using a humidifier and reducing or stopping CL wear if DED is active.

Although we report an increase in DED symptoms among CL wearers, as reported in the literature [30], there was not a significant relationship between CL use and depression among DED patients. This may be attributed to the fact that there are some individual's behaviors such as CL could increase the DED symptoms in DED patients. However, these behaviors have not increased the risk of developing depression.

A limitation of the study was the use of social media to collect responses, and as a result, most of the participants were mainly of the young age group. Although the scales used in our study have been validated $[18,22]$, they are more aimed at screening rather than diagnosis, and therefore, this may overestimate the true prevalence. Participants may have been untruthful and biases cannot be excluded. A larger sample size with the inclusion of other ocular comorbidities can shed light on the psychological impact of ocular pathology on individuals' mental wellbeing.

\section{Conclusions}

In conclusion, suffering from DED is a possible risk factor for developing depression as DED is associated with depression of higher degrees of severity. Depression among DED patients is found to be significantly more prevalent among females and young adult population rather than older adults.

We recommend screening for depression among patients diagnosed with DED in ophthalmology clinics as well as primary care clinics as a proactive measure in improving life quality and to preserve their psychological well-being.

\section{Appendices}




\section{Cureus}

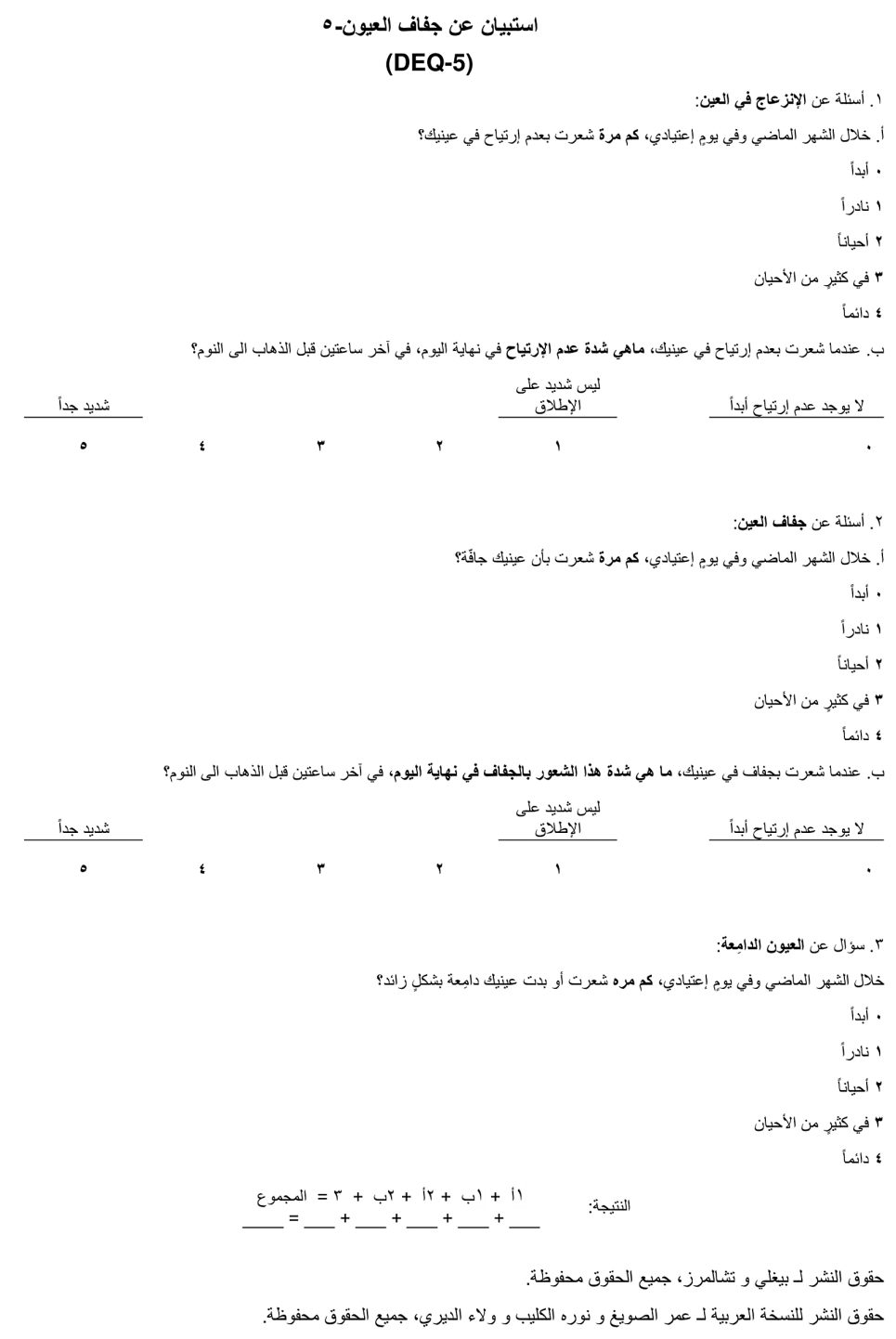

FIGURE 1: Arabic version of DEQ-5

\section{Additional Information}

\section{Disclosures}

Human subjects: Consent was obtained by all participants in this study. Research Ethics Committee of College of Medicine, King Faisal University issued approval 2020-05-13. This study was conducted upon the approval of the research ethics committee of College of Medicine, King Faisal University. All data were confidentially and anonymously collected with consent taken from the participants. Animal subjects: All authors have confirmed that this study did not involve animal subjects or tissue. Conflicts of interest: In compliance with the ICMJE uniform disclosure form, all authors declare the following: Payment/services info: All authors have declared that no financial support was received from any organization for the submitted work. Financial relationships: All authors have declared that they have no financial relationships at present or within the previous three years with any organizations that might have an interest in the submitted work. Other relationships: All authors have declared that there are no other relationships or activities that could appear to have influenced the submitted work.

\section{References}

1. Lemp MA, Baudouin C, Baum J, et al.: The definition and classification of dry eye disease: report of the definition and classification subcommittee of the International Dry Eye WorkShop. Ocul Surf. 2007, 5:75-92. 10.1016/s1542-0124(12)70081-2

2. Stapleton F, Alves M, Bunya VY, et al.: TFOS DEWS II epidemiology report. Ocul Surf. 2017, 15:334-365. 
10.1016/j.jtos.2017.05.003

3. Alshamrani AA, Almousa AS, Almulhim AA, et al.: Prevalence and risk factors of dry eye symptoms in a Saudi Arabian population. Middle East Afr J Ophthalmol. 2017, 24:67-73. 10.4103/meajo.MEAJO_281_16

4. Alharbi AJ, Alanazi NA, Alhamad JR, Alabdulqader RA, Aljamea DA, Alabdulqader SA, Naganathan M: Prevalence of symptomatic dry eye and its risk factors among coastal population in Eastern Province of Saudi Arabia. EC Ophthalmol. 2019, 10:503-509. 10.4172/2155-9570-c6-094

5. Bukhari A, Ajlan R, Alsaggaf H: Prevalence of dry eye in the normal population in Jeddah, Saudi Arabia . Orbit. 2009, 28:392-397. 10.3109/01676830903074095

6. Malhi GS, Mann JJ: Depression. Lancet. 2018, 392:2299-2312. 10.1016/S0140-6736(18)31948-2

7. Alhamyani A, Noor Kalakattawi R, Noor Kalakattawi A, et al.: Prevalence of dry eye symptoms and its risk factors among patients of King Abdulaziz Specialist Hospital (Taif), Saudi Arabia. Saudi J Heal Sci. 2017, 6:140-144. 10.4103/sjhs.sjhs_90_17

8. Al Houssien AO, Al Houssien RO, Al-Hawass A: Magnitude of diabetes and hypertension among patients with dry eye syndrome at a tertiary hospital of Riyadh, Saudi Arabia - a case series. Saudi J Ophthalmol. 2017, 31:91-94. 10.1016/j.sjopt.2017.02.001

9. Mertzanis P, Abetz L, Rajagopalan K, et al.: The relative burden of dry eye in patients' lives: comparisons to a U.S. normative sample. Investig Ophthalmol Vis Sci. 2005, 46:46-50. 10.1167/iovs.03-0915

10. Miljanović B, Dana R, Sullivan DA, Schaumberg DA: Impact of dry eye syndrome on vision-related quality of life. Am J Ophthalmol. 2007, 143:409-415. 10.1016/j.ajo.2006.11.060

11. Na KS, Han K, Park YG, Na C, Joo CK: Depression, stress, quality of life, and dry eye disease in Korean women: a population-based study. Cornea. 2015, 34:733-738. 10.1097/ICO.0000000000000464

12. Jonas JB, Wei W Bin, Xu L, Rietschel M, Streit F, Wang YX: Self-rated depression and eye diseases: the Beijing eye study. PLoS One. 2018, 13:e0202132. 10.1371/journal.pone.0202132

13. Wan KH, Chen LJ, Young AL: Depression and anxiety in dry eye disease: a systematic review and metaanalysis. Eye. 2016, 30:1558-1567. 10.1038/eye.2016.186

14. Hallak JA, Tibrewal S, Jain S: Depressive symptoms in patients with dry eye disease: a case-control study using the beck depression inventory. Cornea. 2015, 34:1545-1550. 10.1097/ICO.0000000000000641

15. Liyue H, Chiang PP, Sung SC, Tong L: Dry eye-related visual blurring and irritative symptoms and their association with depression and anxiety in eye clinic patients. Curr Eye Res. 2016, 41:590-599. 10.3109/02713683.2015.1056804

16. Yilmaz U, Gökler ME, Unsal A: Dry eye disease and depression-anxiety-stress: a hospital-based case control study in Turkey. Pak J Med Sci. 2015, 31:626-631. 10.12669/pjms.313.7091

17. Weatherby TJ, Vasant Raman VR, Agius M: Depression and dry eye disease: a need for an interdisciplinary approach?. Psychiatr Danub. 2019, 31:619-621.

18. Kroenke K, Spitzer RL, Williams JB: The PHQ- 9: validity of a brief depression severity measure . J Gen Intern Med. 2001, 16:606-613. 10.1046/j.1525-1497.2001.016009606.x

19. Zung WW: A self-rating depression scale. Arch Gen Psychiatry. 1965, 12:63-70. 10.1007/978-94-007-07535_104628

20. Lamoureux EL, Tee HW, Pesudovs K, Pallant JF, Keeffe JE, Rees G: Can clinicians use the PHQ-9 to assess depression in people with vision loss?. Optom Vis Sci. 2009, 86:139-145. 10.1097/OPX.0b013e318194eb47

21. Simpson TL, Situ P, Jones LW, Fonn D: Dry eye symptoms assessed by four questionnaires . Optom Vis Sci. 2008, 85:692-699. 10.1097/OPX.0b013e318181ae36

22. Chalmers RL, Begley CG, Caffery B: Validation of the 5-Item Dry Eye Questionnaire (DEQ- 5): discrimination across self-assessed severity and aqueous tear deficient dry eye diagnoses. Contact Lens Anterior Eye. 2010, 33:55-60. 10.1016/j.clae.2009.12.010

23. Al-Qadhi W, Ur Rahman S, Ferwana MS, Abdulmajeed IA: Adult depression screening in Saudi primary care: prevalence, instrument and cost. BMC Psychiatry. 2014, 14:190. 10.1186/1471-244X-14-190

24. Van Der Vaart R, Weaver MA, Lefebvre C, Davis RM: The association between dry eye disease and depression and anxiety in a large population-based study. Am J Ophthalmol. 2015, 159:470-474. 10.1016/j.ajo.2014.11.028

25. Williams DR, González HM, Neighbors H, Nesse R, Abelson JM, Sweetman J, Jackson JS: Prevalence and distribution of major depressive disorder in African Americans, Caribbean blacks, and non-Hispanic whites: results from the National Survey of American Life. Arch Gen Psychiatry. 2007, 64:305-315. 10.1001/archpsyc.64.3.305

26. Akkaya S, Atakan T, Acikalin B, Aksoy S, Ozkurt Y: Effects of long-term computer use on eye dryness . North Clin Istanb. 2018, 5:319-322. 10.14744/nci.2017.54036

27. Hovanesian JA, Shah SS, Maloney RK: Symptoms of dry eye and recurrent erosion syndrome after refractive surgery. J Cataract Refract Surg. 2001, 27:577-584. 10.1016/S0886-3350(00)00835-X

28. Kasetsuwan N, Satitpitakul V, Changul T, Jariyakosol S: Incidence and pattern of dry eye after cataract surgery. PLoS One. 2013, 8:e78657. 10.1371/journal.pone.0078657

29. Shtein RM: Post-LASIK dry eye. Expert Rev Ophthalmol. 2011, 6:575-582. 10.1586/eop.11.56

30. Begley CG, Caffery B, Nichols KK, Chalmers R: Responses of contact lens wearers to a dry eye survey . Optom Vis Sci. 2000, 77:40-46. 10.1097/00006324-200001000-00012 